Archivum, LXX (I), 2020, pp. 165-191

\title{
La literatura en el marco de las neurociencias cognitivas. Nuevas perspectivas de estudio
}

\author{
María Virtudes Núñez Fidalgo \\ INSTITUTO DE INVESTIGACIÓN LINGÜÍSTICA Y LiTERARIA \\ Universidad Autónoma de Santo Domingo \\ mnunez@hotmail.com
}

Recibido: 09/07/2019

Aceptado: 22/10/2019

\section{RESUMEN:}

El trabajo que presentamos surge a partir de una preocupación compartida por un equipo de académicos acerca de las dificultades en el aprendizaje de la lectura de los niños dominicanos, lo que dio como resultado la búsqueda de soluciones basadas en las propuestas más innovadoras de las neurociencias cognitivas que señala Stanislas Dehaene, prestigioso exponente de esta disciplina de estudio.

Esta perspectiva de estudio transdisciplinar nos lleva, por otra parte, a revisar los resultados científicos en torno a la relación entre el hecho literario y la teoría de la mente, con la finalidad de diseñar aplicaciones experimentales en el aula que resulten eficientes y de validación probada. Entre los artículos revisados destacan aquellos que evidencian que la lectura de textos literarios de buena calidad fortalece los procesos cognitivos en la formación de habilidades heterometacognitivas, así como los estudios que se centran en el análisis de la comprensión de la metáfora desde la perspectiva de las neurociencias.

PALABRAS CLAVE: neurociencias cognitivas, investigación literaria, Stanislas Dehaene, aprendizaje lector, estudios de la metáfora. 
Literature in the framework of cognitive neurosciences. The new approaches of study

\begin{abstract}
:
The work we present arises from a concern shared by a team of academics about the difficulties in reading learning of Dominican children, which resulted in the search for solutions based on the most innovative proposals of the cognitive neurosciences that Stanislas Dehaene points out, a prestigious exponent of this discipline of study.

This perspective of transdisciplinary study leads us, on the other hand, to review the scientific results about the relationship between the literary fact and the theory of the mind, with the purpose of designing experimental applications in the classroom that are efficient and of proven validation. Among the reviewed articles stand out those that show that the reading of literary texts of good quality strengthens the cognitive processes in the formation of heterometacognitive skills, as well as the studies that focus on the analysis of the understanding of the metaphor from the perspective of the neurosciences.
\end{abstract} KEY WORDS: cognitive neurosciences, literary research, Stanilas Dehaene, reader learning, metaphor studies.

\title{
1. Introducción
}

Las neurociencias cognitivas forman, junto con la psicología cognitiva experimental, un conjunto de disciplinas transdisciplinares que toman elementos de las ciencias y las humanidades y profundizan en el análisis de objetos de estudio aplicando tecnologías biomédicas, sin desdeñar métodos procedentes de la psicología y la educación. El avance de los descubrimientos en este ámbito es vertiginoso debido a que sus experimentos se realizan con técnicas y equipos de imagenología cerebral que proporcionan imágenes diagnósticas con altos niveles de precisión. Desde nuestra posición filológica, nuestro objetivo consiste en explorar perspectivas de estudio interdisciplinar que puedan concretarse en líneas innovadoras de investigación para el conocimiento del hecho literario y del proceso lector en relación con las funciones del cerebro humano.

Los informes de neurociencias cognitivas o psicología cognitiva experimental exponen los descubrimientos en torno a los 
procesos de la adquisición del lenguaje y el aprendizaje de la lectura. Existen evidencias en los estudios experimentales de laboratorio que colocan la interpretación de las expresiones comunicativas, lingüísticas y literarias en una nueva dimensión gnoseológica. Los especialistas visualizan e interpretan las operaciones del cerebro utilizando procedimientos tomados de las ciencias básicas como matemáticas y física y de las ciencias aplicadas como psicología y neurociencias. Por otra parte, muchas de las preguntas de estudio e hipótesis sobre las cuales establecen sus investigaciones se sitúan en el marco de la psicología y las ciencias sociales.

Nuestra propuesta tiene su origen en la búsqueda de soluciones que se precisan para resolver situaciones propias de la experiencia cotidiana en las aulas dominicanas. Entre ellas, nos preocupa un problema socioeducativo que dificulta el desarrollo escolar de los niños y adolescentes de manera dramática: las limitaciones en el proceso de comprensión y expresión en lectura y escritura. De ahí surgió la necesidad de profundizar en la relación de la lectura y la literatura con las funciones del cerebro. Nos propusimos introducirnos en las teorías más avanzadas, aquellas que con evidencias científicas explican cuáles son los mejores métodos para el aprendizaje de la lectoescritura.

Los descubrimientos de Stanislas Dehaene, experto en las bases cerebrales aplicadas a las operaciones cognitivas en los distintos estadios del desarrollo humano, resultan de gran interés para nosotros porque muestran con evidencias científicas la vinculación entre las funciones del cerebro y el desarrollo lector, así como los cambios que se producen en el cerebro durante el proceso de aprendizaje, tanto en niños como en adultos.

En segundo lugar, los estudios en torno a la función del texto literario y la teoría de la mente, permiten realizar aplicaciones experimentales en el aula con la consistencia metodológica que exigen los protocolos de investigación. Los hallazgos publicados por investigadores norteamericanos permiten sustentar, también con evidencias científicas, que la lectura de textos literarios de 
buena calidad fortalece los procesos cognitivos en la formación de habilidades heterometacognitivas (Kidd y Castano, 2013). Con la ejecución de estas habilidades se promueve el pensamiento reflexivo, crítico y creativo que tanto se recomienda desde la perspectiva constructivista y que se ha convertido en un reto para la educación en la sociedad de la comunicación global del siglo xxI.

El estudio de las habilidades metapragmáticas y metacognitivas en la ejecución y comprensión de relatos constituye también un interesante campo de estudio. Un trabajo de especial interés fue el que se realizó con estudiantes de nivel primario en Gerona, donde se evidencia que el uso del lenguaje metafórico contribuye a mejorar las competencias metacognitivas en los niños (Rodríguez y Gallart, 2014).

Por último, presentamos los resultados de estudios recientes donde se analiza la comprensión de la metáfora desde la perspectiva de las neurociencias. En síntesis, pensamos que esta exploración interdisciplinar puede contribuir a fortalecer de la plataforma teórica de la investigación filológica y promueve el conocimiento de las funciones del hecho literario en el desarrollo del cerebro humano.

\section{Un problema de estudio: la lectura en los niños y jóvenes dominicanos}

La República Dominicana es un país emblemático en la historia de Occidente por ser el primer enclave hispano de América, donde se fundó la primera universidad y se escribieron los primeros textos literarios americanos. Con el desarrollo del sistema educativo oficial y la aparición de la Escuela Normal, la educación dominicana está superando grandes retos, como sucede con la inclusión de todos los niños en el sistema escolar. Sin embargo, existe un problema acuciante de acceso a la lectura que dificulta el progreso de los ciudadanos y condiciona las posibilidades para el futuro de las nuevas generaciones.

La situación deficitaria en las mediciones de competencia lectora que evidencian las evaluaciones internacionales (SERCE, 
TERCE, PISA) supone para los docentes un desafío con el que todos nos sentimos comprometidos, desde los maestros de primaria hasta los profesores de enseñanza superior (Valdés et al., 2008; UNESCO/OREALC, 2015; Flotts et al., 2015; Bos et al., 2016).

En reiteradas ocasiones se refleja esta cruda realidad en informes que no dejan lugar a dudas. Veamos, como ejemplo, el siguiente comentario descriptivo del BID, en relación con los resultados de las pruebas PISA:

De los 72 países que participaron en el Programa para la Evaluación Internacional de Alumnos (PISA), aplicada en 2015, los diez países latinoamericanos y caribeños se ubicaron en el tercio inferior de la distribución. En los rankings de las tres materias (lectura, matemática y ciencia), Chile encabeza la lista de los países de la región y Republica Dominicana en la última. Los aprendizajes son bajos, con respecto al promedio de la OCDE, la región está rezagada en más de 2,5 años de escolaridad. (BID Educación, 2016, 6)

Los problemas de la lectura afectan irremediablemente a la aplicación del texto literario en las aulas. En la universidad pública dominicana, la mayor dificultad para el acceso a la comprensión del texto literario consiste en los problemas para acceder a los niveles inferencial y crítico del texto. Se trata de un problema de fondo, con múltiples aristas como la falta de hábitos de lectura y el elevado porcentaje de niños y adolescentes que son víctimas del analfabetismo funcional y global.

Los docentes que trabajamos en las aulas impartiendo cursos de grado y posgrado sabemos que cuando no se resuelven los problemas de lectura y escritura en los ciclos de primaria y secundaria se produce un notable atraso en el desarrollo curricular de los estudiantes universitarios, disminuyendo sus posibilidades de adquisición de conocimientos, e impactando la eficiencia profesional de los mismos, una vez que concluyan sus estudios de grado.

La didáctica de la literatura no resulta ajena a este problema. Si bien el desarrollo del proceso lector es un asunto complejo y 
de carácter multifactorial, la ausencia de la lectura literaria en las aulas podría arrojar algunas explicaciones. De hecho, la disminución del espacio de enseñanza literaria en este país en primaria y secundaria se ha acrecentado por la exigencia oficial de discriminación de la literatura como parte de la aplicación del nuevo diseño curricular vigente desde 2017. Apenas se encuentran algunas unidades dentro de un cúmulo de contenidos de lengua española que, por otra parte, anulan el aprendizaje secuenciado de los temas gramaticales, a pesar de que, como es bien sabido, forman parte esencial en la construcción de la arquitectura del texto escrito.

De este modo, el nuevo diseño curricular que se aplica en todas las escuelas de manera rigurosa y bajo cuyo mandato se regula la planificación los ciclos de formación escolar previos a la universidad, no contempla la impartición de la materia de literatura en ningún curso de primaria, secundaria, ni bachillerato. Se elimina el estudio secuenciado de la historia de la literatura en cualquiera de sus notaciones, ya sea hispánica, latinoamericana, universal e incluso dominicana. La reducción de los contenidos de literatura implica dejar al descubierto algunos temas que permanecen pero quedan inconexos, porque han sido tomados de forma aleatoria desde la historia de la literatura, la retórica y estilística literaria. En una planificación que se define como textual, funcional y comunicativa, la literatura se relega a escasas unidades carentes de ordenación sistemática o estructural (MINERD, 2016a; 2016b).

Para intentar cambiar esta realidad, y ante la preocupación sostenida para mejorar el aprendizaje lector en este país, un grupo de profesores e intelectuales (historiadores, educadores, matemáticos, psicólogos, filólogos, escritores) nos hemos organizado para profundizar en el estudio del proceso lector. El diagnóstico situacional de la lectura y escritura sobre todo en el primer ciclo de primaria nos llevó a transitar los caminos de la discusión mediática en la prensa dominicana. En el análisis sobre los métodos de aprendizaje de la lectura encontramos la mejor respuesta den- 
tro de los avances que proporcionan las neurociencias aplicadas a la lectura, porque aportan argumentos innovadores basados en métodos científicos y resultados obtenidos con aplicaciones tecnológicas de última generación. Los argumentos que aporta esta disciplina para el conocimiento del proceso lector superan las interpretaciones teóricas clásicas, corrigen y enriquecen los planteamientos procedentes de la observación pedagógica externa.

Desde hace algunos años, se están dando experiencias interesantes en la educación dominicana para introducir a los docentes en el conocimiento de las neurociencias aplicadas a la educación. Uno de los pioneros en ese ámbito es el doctor Emilio García, de la Universidad Complutense de Madrid, quien ha impartido cursos y talleres organizados por el Ministerio de Educación Superior, Ciencia y Tecnología. Entre sus aportes destaca también la dirección de la primera tesis de neuroeducación y lingüística de un profesor dominicano, el doctor Carlisle González Tapia (2016).

Otra experiencia relevante en ese sentido fue la organización de la agenda para la divulgación de las teorías del Dr. Dehaene promovidas por un grupo de profesores e intelectuales dominicanos desde el año 2017 hasta la actualidad. Se organizaron diversas actividades para mostrar sus hallazgos en torno al aprendizaje de la lectura y evidenciar cuál es el método más eficiente para el aprendizaje de la lectura desde la posición de las neurociencias cognitivas, así como los principios del aprendizaje efectivo que deben aplicarse en las aulas, con el fin de que los estudiantes puedan optimizar su tiempo de estudio y ampliar el efecto de su aprendizaje.

Un momento culminante de estas actividades se dio con la visita del profesor Dehaene y la presentación de dos conferencias como parte del programa del Collège de France. La primera de ellas, Ciencias cognitivas y educación: los grandes principios del aprendizaje, propone las bases fundamentales del aprendizaje a la luz de los descubrimientos de la neurociencia y la segunda, Cerebro, lectura y alfabetización, constituye una síntesis de sus investigacio- 
nes sobre las funciones del cerebro en el proceso de alfabetización. Por su parte, la Dra. Ghislaine Dehaene-Lambertz presentó una conferencia magistral sobre las bases cerebrales en la adquisición de la lengua en los recién nacidos. Ambas disertaciones tuvieron mucho eco en los medios dominicanos (Castro, 2017).

\section{La teoría del reciclaje neuronal de la lectura de S. Dehaene}

Probablemente, el investigador francés Stanislas Dehaene es el científico europeo de mayor prestigio en torno al estudio neurocientífico de la lectura, durante las dos primeras décadas del siglo xxi. Basta con ver el impresionante nivel de producción de artículos científicos en la página del Collège de France (s. f.), para comprobar la relevancia de sus investigaciones a nivel mundial. Sus teorías se enriquecen con los aportes de su esposa, la doctora en pediatría Ghislaine Dehaene-Lambertz, que investiga aspectos relacionados con la emergencia del lenguaje en recién nacidos y el desarrollo de prelectura en niños pequeños.

El cerebro lector. Últimas noticias sobre neurociencias de la lectu$r a$, la enseñanza, el aprendizaje y la dislexia es una obra reveladora para nosotros. Salió a la luz en 2009 y fue traducida al español en 2011, en la colección Ciencia que Ladra de la editorial argentina Siglo XXI, donde se reúnen obras de divulgación escritas por los propios científicos (Dehaene, 2009). La cuarta edición que hemos consultado para este trabajo es la más actualizada (Dehaene, 2017).

Además de mostrar un exhaustivo y actualizado acopio bibliográfico sobre el tema, el autor recopila varias décadas de trabajo en el laboratorio de Neurospin de París, realizadas con técnicas de imagenología funcional y estructural (escáner cerebral, imágenes por resonancia magnética funcional, tomografías por emisión de positrones) para conocer la compleja arquitectura de la cognición humana. A través de la tecnología de neuroimágenes se proporcionan retratos de la anatomía cerebral y se muestra la distribución de las redes neuronales para explicar que aprender a leer introduce modificaciones muy profundas en la 
anatomía y en la actividad cerebral todo a lo largo de la cadena que enlaza la visión con el lenguaje hablado.

La realización y comparación de mapas del cerebro en los distintos estadios de aprendizaje, examinando la evolución de las redes neuronales desde la etapa analfabeta hasta la alfabetización, le permite presentar datos para informarnos sobre las patologías del desarrollo o adquiridas relacionadas con la lectura y ofrecer estrategias pedagógicas basadas en evidencias neurocientíficas para mejorar las buenas prácticas docentes.

En el cerebro lector, el autor introduce una discusión central en la investigación sobre lectura cuando se pregunta cómo accede el cerebro al significado de las palabras, si se accede directamente al significado de las palabras o si primero se convierten las letras en sonidos y luego estos últimos nos permiten acceder al significado. S. Dehaene resuelve esta discusión a partir del análisis del modelo de dos rutas para la lectura, basándose en teorías de psicología cognitiva, evidencias clínicas, estudios de lesionados cerebrales aléxicos e investigaciones realizadas con niños.

Se postula una ruta fonológica que se apoya en la recodificación fonológica para acceder al significado y una ruta léxica que permite acceder directamente desde la cadena de letras al significado almacenado en el léxico mental. Se propone que ambas rutas están activas en el adulto. En los lectores entrenados se daría un uso prevalente de la ruta directa, mientras que el uso de la ruta fonológica sería predominante durante el aprendizaje de la lectura. La evidencia de este modelo de doble ruta a través de imagenología cerebral está determinado por la existencia de dos redes separadas, una que se dedica al procesamiento del sonido y otra al del significado, que permite el acceso al léxico mental. Las dos rutas se ponen en juego en todas las lenguas sin importar las características ortográficas y se hallan localizadas en áreas similares del cerebro. La diferencia se encuentra en el modo en que se activan las distintas áreas del cerebro en cada lengua dependiendo de su transparencia y granularidad: en las lenguas alfabéticas transparentes como el español y el italiano, 
habría una mayor activación del área auditiva, mientras que al leer caracteres chinos se produce la mayor activación de las áreas relacionadas con el léxico mental.

Estudios realizados con lesionados cerebrales que sufren alexia evidencian la presencia de lo que el autor llama "caja de letras del cerebro", un área encargada del procesamiento de la forma visual de las palabras. La localización del área de la caja de letras (al borde de la cisura témporo-occipital izquierda) es prácticamente la misma para todos los sujetos y se activa durante la lectura en los cerebros de los lectores de todas las lenguas: español, francés, inglés, chino y japonés, por citar algunas. Las evidencias presentadas le permiten a Dehaene hablar de la universalidad de los circuitos neuronales involucrados en la lectura y explicar las modificaciones en la especialización de determinadas áreas que estaban dedicadas a la visualización de imágenes para destinarlas a la lectura, como parte del proceso educativo del aprendizaje de esta habilidad tan importante en el desarrollo del género humano (Dehaene, 2017, 145-152).

\subsection{Funciones de la literatura en el aprendizaje lector}

El inicio infantil de la lectura es previo al aprendizaje formal y comienza con la etapa logográfica o pictórica, en la que los niños hacen el reconocimiento visual de palabras como si fueran objetos o dibujos (color, forma, tipografía...). En esta fase no se lee en sentido estricto, sino que el sujeto intenta establecer relaciones entre la forma de la palabra y su significado, sin prestar atención a las letras que la componen ni establecer relaciones entre estas y los sonidos que las representan. Esta habilidad, poder segmentar las palabras en sus constituyentes (grafemas) y establecer relaciones entre estos y los sonidos que los representan en el habla (fonemas), es el mecanismo que rige durante la segunda etapa, la etapa fonológica en la que el niño aprende los mecanismos de conversión y pone en juego las habilidades de conciencia fonológica, esenciales para el aprendizaje de la lectura. Finalmente, la etapa ortográfica, se caracteriza porque en el cerebro del lector 
se ha ido formando un léxico mental que contiene unidades de distinto tamaño y que incluye información sobre la frecuencia de las diferentes unidades y de sus vecinos.

Los hallazgos en torno al aprendizaje de la lectura le permiten a Dehaene comparar la eficacia de los principales métodos de lectoescritura: el método fónico y método global o de lenguaje integrado. La raíz de la discusión de los métodos de lectoescritura está en definir cuál es la unidad en la que hay que basar la enseñanza durante el inicio de proceso de alfabetización: el fonema (y la enseñanza sistemática de la relación grafema/fonema) o la palabra completa. El autor presenta evidencias empíricas que señalan que el método fónico es más favorable, ya que les brinda a los niños una herramienta de autoaprendizaje que les permitirá leer todas las palabras que se les presenten. Asimismo, basándose en evidencias de resultados experimentales, afirma que es conveniente trabajar tempranamente las habilidades de conciencia fonológica con lo que podríamos definir como ejercicios literarios adaptados al nivel de los infantes:

Para el niño de nivel inicial (es decir, preescolar, jardín de infantes o Kindergarten), esta descomposición en fonemas no es para nada evidente. El aprendizaje de la lectura en un sistema ortográfico alfabético es lo que la hace salir a la luz. La aparición de la conciencia fonológica es una de las etapas clave en el camino de la lectura. Sin embargo, la investigación muestra que puede acelerarse su adquisición si se realizan juegos lingüísticos desde edades tempranas. Las canciones o rondas infantiles, las rimas, las jitanjáforas ("Al soldado, dado" o, en un verso de Vicente Huidobro, "uluLAyo uluLAmento") las adivinanzas ("Es cara y termina bajo. / Lo adivino sin trabajo"), la búsqueda de palabras que terminen con determinado sonido o de otras que están ocultas en una frase ("Oro parece, / plata no es..."), etc.: todo lo que suponga manipular los sonidos de las palabras prepara a los niños para la lectura. (Dehaene, 2015, 44-45) 
He aquí una de las funciones primordiales de la literatura escrita en el desarrollo escolar: contribuir a que los niños sean conscientes de que están manejando letras, sílabas, sonidos, y que con esta toma de conciencia fonológica puedan avanzar en la combinación y manejo de unidades de mayor complejidad: las palabras, los enunciados y las oraciones. La lectura constituye un sistema artificial que debe ser aprendido por el cerebro y la literatura contribuye a darle sentido, a insertar los elementos del sistema de forma rápida, permanente y profunda. Las canciones, la poesía, la dramatización, el juego literario generan entornos amigables, lúdicos, permite a los niños mantener el interés por seguir aprendiendo, sin importar el enorme esfuerzo que para ellos supone recordar la forma y la dinámica combinatoria de signos que, en principio, no necesitan para manejarse en su vida cotidiana, puesto que el espacio vital de su actividad lingüística está muy bien desarrollado con la expresión y comprensión oral.

En el caso de que pueda acceder a textos infantiles y juveniles de calidad, el niño va a encontrar en el camino de la lectura literaria, elementos de sorpresa, de observación y reflexión que le permiten regular sus emociones y sentimientos a partir de la mirada atenta a las conductas de los personajes. Se introduce entonces en un conocimiento del mundo que genera una serie de habilidades metacognitivas, de planificación y adaptación y aprende a regular conductas, ensaya su capacidad de decisión, rechazando o asumiendo situaciones que de otro modo le resultarían incomprensibles o muy difíciles de gestionar. Todo ello le permite enfrentar con cierto equilibrio psicológico, realidades vitales insospechadas y a veces, crueles (la enfermedad, la pobreza, la violencia, la muerte).

Las neuronas espejo y la teoría de la mente fundamentan la base de esta contribución. Otros autores mencionan la funcionalidad del arte para facilitar la capacidad de reserva cognitiva, es decir, a mayor capacidad de reserva cognitiva también se genera una mayor resistencia a los efectos de procesos patológicos agu- 
dos y subagudos (traumatismos cerebrales, trastornos neurodegenerativos, demencias) mitigando y disminuyendo los daños que puedan ocasionar durante largo tiempo (Fornazzari, 2008; Gómez Higuera, 2015).

S. Dehaene defiende siete grandes principios de aprendizaje, muchos de los cuales los conocen los docentes, pero a veces dejan de aplicarse por motivos ajenos a la escuela o a los maestros. Entre ellos, destacamos el principio de compromiso activo, de atención y disfrute, puesto que concierne a los factores que inciden en la velocidad del aprendizaje y la fuerza con la que se retiene la información en la memoria. Este principio incide directamente en el ejercicio literario como actividad lúdica y creativa: el maestro debe proponer un contexto inspirador que haga que el niño siga activo, sienta alegría al aprender, se sienta autorizado a cometer errores y al mismo tiempo sea corregido cuando corresponda y recompensado por sus esfuerzos. Las actividades deben ser lúdicas y apelar, por ejemplo, a juegos de rimas, rondas, canciones de tipo "acumulativo", jitanjáforas o "ecos", "palabras imposibles" o "inventadas", los dominós o naipes de grafemas, juegos de improvisación al estilo de los romances, las coplas y las décimas tradicionales (o el freestyle afín al hip hop), búsqueda de "palabras ocultas", breves pregones, etc. Las actividades deben también estimular la participación y la creatividad de los niños (Dehaene, 2017, 93-94).

En definitiva, podemos decir que se conocen los pasos necesarios para obtener los mayores niveles de eficiencia en el aprendizaje y desarrollo del proceso lector y en el desarrollo educativo de los estudiantes. Sin embargo, nos corresponde a nosotros, como docentes, ver de qué manera podemos aplicar esas teorías en el aula, para conseguir que los estudiantes no sólo aprendan, sino que conviertan la experiencia de la lectura en fuente de enriquecimiento y reflexión permanente, para promover diseños y soluciones creativas a los viejos problemas que esperan por una solución real. 


\subsection{Del Homo sapiens al Homo docens: aprendizaje y crea- tividad}

Además de sus hallazgos sobre los métodos de lectoescritura, el autor propone la hipótesis de que el cerebro humano no evolucionó para la lectura y la escritura, sino que fue la escritura la que se adaptó a nuestro cerebro. Asimismo, sostiene que la escritura generó una revolución cultural y permitió ampliar nuestras habilidades cognitivas, pero que no es el único constructo cultural restringido por la arquitectura del cerebro y que debería ser posible conectar otras actividades humanas con mecanismos cerebrales. En línea con esto último, el autor reseña una serie de investigaciones sobre música, religión, matemáticas, arte y ciencia que tratan de identificar los precursores y circuitos cerebrales asociados con ellas.

Dehaene se refiere al Homo docens para definir lo que hace exclusivo al género humano: la curiosidad o motivación hacia el descubrimiento y la extraordinaria flexibilidad cerebral de aprendizaje para formular hipótesis y seleccionarlas en función de su adecuación con el mundo exterior hasta transformarlas en conocimientos estables. La curiosidad lleva a la invención a través de la experimentación durante el proceso de aprendizaje; la evolución humana es una línea constante y continua de invenciones que se modifican a lo largo del tiempo y cambian el modo de hacer y de ser. Esto sucede desde los tiempos antiguos hasta el momento actual, y el texto literario de buena calidad se convierte en un hecho social atrayente que promueve el desarrollo de las sociedades para reflexionar sobre el pasado, evaluar el presente y proyectar el futuro dentro de un marco estético placentero y motivador para los lectores:

Cuando leemos una poesía o una novela, si el escritor está bien dotado al escribir, nosotros revivimos literalmente, en nuestros circuitos neuronales los acontecimientos pasados, presentes o futuros, imaginarios o reales que el autor ha querido compartir con nosotros, y cuando leemos un libro de Matemáticas o de Ciencias, re- 
construyendo en nuestra cabeza las ideas del autor, argumentamos el poder de representación de nuestro propio cerebro. (Dehaene, 2018, 50)

Su hipótesis de reciclaje neuronal se vincula con la modelación de los procesos de creatividad, que se evidencian a partir de los límites establecidos en los sistemas de escritura. Apoyándose en los resultados de sus propias investigaciones y coincidiendo con especialistas de distintas áreas como Levi-Strauss, Sperber, Chomsky y Brown, el autor considera la existencia de sistemas culturales compartidos universalmente y propone como evidencia el hecho de que todos los sistemas de escritura comparten una gran cantidad de rasgos:

La hipótesis de reciclaje neuronal predice que la creatividad humana está atada a la arquitectura cerebral, una idea que se opone con claridad al relativismo cultural, que considera que las variaciones culturales son en esencia ilimitadas. Si hay alguna verdad en el reciclaje neuronal, nuestra constitución genética refrena drásticamente el conjunto de sistemas de escritura que pueden aprenderse. Esta predicción nos lleva a cuestionar la aparente falta de límites de la diversidad cultural. Al rasgar la cara externa de las variaciones culturales superficiales más obvias, deberíamos descubrir evidencia de estructuras profundas compartidas universalmente. (Dehaene, 2018, 213)

\section{Una línea de estudio prometedora: lectura literaria y teoría de la mente}

El estudio de la literatura en el siglo xxi deberá asumir el desafío de los descubrimientos de las neurociencias, en tanto que es en la mente del autor y del lector donde se generan las ideas y la expresión estética como producto individual. En este ámbito, la literatura es arte y el arte ha sido definido por Luis Fornazzari como un producto del cerebro humano que evolutivamente ha desarrollado sus vías neuronales específicas. El producto de la 
creatividad particular del artista se integra en una expresión final provocando una explosión de elementos neurosociales y psicológicos (Fornazzari, 2008, 154). Todos sabemos que el alcance de la obra de arte literaria trasciende al lector. Una poesía, un cuento, una obra de teatro puede proyectarse en el tiempo y en el espacio, convertirse en un símbolo cultural del que disfrutan durante siglos distintas sociedades.

De algún modo, esta propuesta se relaciona con la reflexión de Dehaene sobre la evolución del género humano y lo que hace única a nuestra especie entre los seres vivos:

La originalidad de nuestra especie puede venir de una combinación de dos factores: una teoría de la mente (la habilidad para imaginar la mente de los otros) y un espacio de trabajo global consciente (un retén interno donde puede volver a combinarse una infinita variedad de ideas). Ambos mecanismos, inscriptos en nuestros genes, conspiran para hacernos la única especie cultural. (Dehaene, 2017, 23)

El concepto de 'teoría de la mente' (ToM) identifica la habilidad para comprender y predecir la conducta de otras personas, sus conocimientos, sus intenciones y sus creencias. Se define también como una habilidad heterometacognitiva, es decir, cómo un sistema cognitivo logra conocer los contenidos de otro sistema cognitivo diferente de aquel con el que se lleva a cabo dicho conocimiento (Tirapu-Ustarruz et al., 2007). Esta teoría surgió a finales de los setenta del siglo pasado con los experimentos pioneros con simios de Premack y Woodruf y resulta especialmente interesante en la comprensión de enfermedades como el autismo y el síndrome de Asperger, donde los niños experimentan serias dificultades en procesos de interacción comunicativa (Premack y Wodruff, 1978). En 2013, investigadores norteamericanos mostraron los resultados de experimentos donde se evidencia que la lectura de textos literarios de calidad fortalece las habilidades relacionadas con la "teoría de la mente" (Kidd y Castano, 2013). 
Los autores describen cinco experimentos que demuestran que la lectura de ficción literaria procedente de autores finalistas del National Book Award condujo a un mejor rendimiento en las pruebas de ToM afectiva y cognitiva, en comparación con la lectura de no ficción, de ficción popular (best sellers de Amazon) o de nada en absoluto.

Estos autores se apoyan en antecedentes que demuestran que la lectura de ficción es una práctica cultural muy útil para promover y refinar la sensibilidad interpersonal durante toda la vida, sobre todo por el aumento de la empatía autodeclarada y porque contribuye a expandir nuestro conocimiento de las vidas de los otros, ayudándonos a reconocer nuestras similitudes con los demás. Sin embargo, no es cualquier tipo de texto el que promueve los cambios en la teoría de la mente, sino que solo la ficción literaria de calidad obliga al lector a una especie de compromiso con los procesos de ToM. En su artículo, nos muestran que leer literatura de buena calidad no solo mejora nuestra capacidad de empatía con los demás, sino que fortalece procesos de pensamiento que nos permiten enfrentar las relaciones sociales complejas, como las que intervienen en el acto de entender el pensamiento y las emociones de otros.

Partiendo de la teoría literaria de D. Miall, D. Kukin, R. Barthes y M. Bajtín y de la teoría cognitiva de Bruner, los investigadores toman conceptos como el de 'polifonía' y explican que el texto literario en su forma escritural y sus contenidos constituye un desafío para el lector a partir del extrañamiento o desfamiliarización lingüística y estilística. La ficción pone en movimiento las habilidades de ToM de una manera única, puesto que activa procesos psicológicos que dan acceso a conocer las experiencias subjetivas de los personajes. Así como sucede en la vida real, el universo de la ficción está lleno de situaciones vitales complicadas y difíciles de discernir sin la adecuada exploración y reflexión sobre los acontecimientos que se relatan.

La esencia no real de la ficción permite que el lector se involucre las situaciones de los personajes sin vivir el riesgo que 
esas experiencias provocarían en la vida real y, por tanto, sin enfrentar las consecuencias de esas vivencias, pero conociéndolas como si las hubiera experimentado. Hay también una diferencia sustancial entre la literatura de ficción de calidad y los best sellers. Según estos investigadores, la ficción de calidad trastorna las expectativas del lector en torno al argumento y las acciones actanciales, exigiéndole reconfigurar el mapa de predicciones sobre el texto y, por tanto, poner en marcha los procesos de la ToM. En el caso de los best sellers, las acciones de los personajes son más predictibles y esto hace que no se active la ToM. Sin embargo, en la ficción literaria de calidad se produce a menudo el quiebre o la disrupción de las expectativas del lector con respecto a las posibles acciones de los personajes a lo largo de la historia, con lo cual se activan los procesos de la ToM, contribuyendo al desarrollo de una conciencia crítica activa.

Es importante añadir que los resultados estadísticos de esta investigación no arrojan informaciones cuantificables que relacionen las habilidades ToM con la literariedad del texto de ficción, por tanto, se necesita continuar investigando sobre este aspecto.

Curiosamente, al igual que está sucediendo ahora en la República Dominicana, donde prácticamente se anula la enseñanza de la literatura en la formación escolar, estos autores previenen a la comunidad educativa contra la opacidad del aprendizaje de la literatura en la planificación del currículo de la enseñanza formal norteamericana y asignan un papel significativo al aprendizaje de la literatura para promover habilidades profundas de pensamiento e interacción social.

Definitivamente, la lectura de textos de ficción es una actividad cerebral que va mucho más allá del mero entretenimiento, y la construcción de un marco conceptual en torno al tema está resultando de gran importancia para investigadores españoles en teoría literaria. Rafael Núñez Ramos, de la Universidad de Oviedo, ha publicado varios trabajos donde se aborda la fundamentación teórica del pensamiento narrativo en relación con los 
procesos cerebrales y mentales movilizados en la lectura de textos literarios, partiendo de que la literatura es una manifestación artística que involucra la expresión humana del sentimiento en un sentido amplio, desde las sensaciones hasta la visión del mundo y la comprensión de la sociedad en la que se desarrolla el individuo (Núñez Ramos, 2010; Alonso García, 2011). En cierto sentido esta perspectiva de estudio coincide con la propuesta desde la Universidad de Valladolid por Martín Jiménez (2014); en su ensayo acerca de la relación entre la retórica clásica y la neurociencia actual recupera la visión aristotélica de las emociones como movimientos del alma que se expresan a través de los gestos corporales y de ahí surge la noción latina de "moción de sentimientos", destinada a "mover" emocionalmente a los receptores.

Un concepto que propone Núñez Ramos (2017) y que resulta fructífero en la construcción de un nuevo marco transdisciplinar para los estudios literarios es el de la neuroestética, que consiste en descubrir dentro de la actividad de las neuronas y el cerebro lo que define la experiencia íntima en la que la obra de arte actualiza sus potenciales y se constituye como tal. Si bien la neuroestética aplicada a la literatura es un concepto que se encuentra en proceso de análisis y discusión, el carácter de aporte al conocimiento transdisciplinar en la teoría literaria se evidencia desde la propia definición y aplicación de ese término.

\section{Investigaciones sobre habilidades metapragmáticas y meta- cognitivas en la construcción de relatos}

La conciencia metapragmática forma parte de las habilidades metacognitivas y metalingüísticas, entendiéndolas no en el sentido de Roman Jakobson, sino de cognición acerca del lenguaje, es decir, el control consciente que cada individuo ejerce sobre su propia lengua. El concepto metapragmático se define como la capacidad que posee un sujeto para establecer relaciones entre un mensaje lingüístico oral y su contexto extralingüístico.

En un estudio aplicado a estudiantes entre 8 y 9 años a los que se invitaba a escribir un relato para mejorar el desarrollo de 
la expresión escrita, se evidenció una correlación positiva, lineal y significativa entre la conciencia metapragmática y la memoria operativa, concluyendo que el incremento de la conciencia metapragmática no depende solo del nivel lingüístico del individuo sino también de una maduración de los sistemas cognitivos involucrados en el almacenamiento y procesamiento de la información (Crespo et al., 2010). Establecer relaciones entre un mensaje lingüístico oral y su contexto de uso implica el ajuste del emisor o destinatario con los parámetros contextuales donde se produce el mensaje y esta habilidad le permite al hablante gestionar usos sofisticados del lenguaje, mostrando así un dominio creciente del sistema lingüístico y sus convenciones de uso.

Resulta para nosotros de especial interés la reflexión que se hace en este trabajo sobre la llamada situación retórica que se define como una representación mental que guía la escritura del sujeto y se compone de tres elementos básicos: el tópico, los lectores y el propósito. La escritura de un texto literario ha de responder siempre a una situación retórica, aunque hay cierta discusión entre los elementos de que la componen. En las conclusiones de su estudio, los investigadores marcan una relación directa entre la conciencia metapragmática y la calidad de la producción de discurso escrito en los niños evaluados. Esa relación es directamente proporcional, es decir, aquellos niños que tenían un mejor desempeño en escritura, fueron también los que dieron mejores respuestas metapragmáticas (Crespo et al., 2010, 198).

En cuanto a las habilidades metacognitivas, vamos a referirnos a resultados de estudios recientes que exploran las relaciones entre metáfora y metacognición en los niveles de escolarización primaria, evidenciando que el uso del lenguaje metafórico promueve la metacognición en los niños.

En una intervención realizada con niños de educación infantil de la Escuela Pública de Girona, se demostró que el lenguaje metafórico posibilita a los alumnos reflexionar sobre cómo gestionan su actividad mental y ganar autonomía en su proceso de aprendizaje (Rodríguez y Gallart, 2014). Los investigadores ex- 
plican que la metáfora facilita la estimulación de la metacognición porque los estudiantes toman conciencia de cuál es el objetivo de cada tarea, de sus propias estrategias y de la importancia de revisar el proceso. La metáfora ayuda a cada niño en la gestión de sus emociones y resulta una herramienta muy útil para resolver los conflictos que surgen en el aula.

\section{Estudios innovadores acerca del procesamiento de la metá- fora}

El estudio de mayor relevancia sobre la comprensión de la metáfora desde la perspectiva neurocientífica fue publicado por un equipo de investigadores del Centro de Investigación Multidisciplinaria del Cerebro de la universidad israelí de Bar-Ilan. Este equipo ha estudiado durante dos décadas el procesamiento cerebral y cognitivo de las metáforas, comparando individuos normales y enfermos, en lo que se refiere al procesamiento de las metáforas novedosas, como las que pueden extraerse de la poesía, con las metáforas convencionales, las expresiones literales y con expresiones de parejas de palabras sin sentido ni relación. Los resultados evidencian que los individuos neurotípicos manejan con flexibilidad la gama completa de relaciones semánticas expresadas en el lenguaje humano, incluidas las relaciones metafóricas (Faust y Kenett, 2014).

Los investigadores dicen que esta notable capacidad semántica puede asociarse con patrones distintos y flexibles de interacción hemisférica, incluida la participación del hemisferio superior derecho para procesar nuevas metáforas. Sin embargo, puede verse afectada en condiciones clínicas específicas (síndrome de Asperger y esquizofrenia). En el informe se interpretan las diferencias individuales utilizando los términos rigidez, caos e integración, que sirven para describir los patrones de los estados de las redes de memoria semántica que conducen a un bienestar semántico en los individuos normales o lo interrumpen en los enfermos. Este continuo incluye estados de memoria semántica demasiado rígida y caótica en personas enfermas que 
contrastan con la flexibilidad semántica de las personas normales. Entre los dos extremos se encuentran diferentes estados de la estructura de la memoria semántica relacionados con las diferencias individuales en la creatividad semántica. Los investigadores sugieren que el procesamiento semántico eficiente se logra en ambos hemisferios cerebrales mediante la integración semántica, un equilibrio entre la rigidez semántica y el caos semántico. Cuando un hemisferio domina al otro, puede llevar a la rigidez semántica o al caos semántico, alejándose de la integración semántica y, por lo tanto, impidiendo el procesamiento del lenguaje metafórico.

En su más reciente estudio, partiendo de hallazgos anteriores en relación con la comprensión de la metáfora, la memoria semántica y la creatividad, estos investigadores han comparado el procesamiento de la metáfora en individuos con niveles creativos inferiores y superiores (Kenett et al., 2018). En sus conclusiones, exponen que el principal hallazgo consistió en que los individuos creativos superiores fueron más rápidos en la comprensión de metáforas convencionales y novedosas, en comparación con los individuos creativos inferiores. Además, los individuos creativos superiores eran significativamente más precisos en la comprensión de metáforas novedosas.

\section{A modo de conclusión: una mirada al futuro de las relaciones entre los estudios filológicos y las neurociencias cognitivas}

En nuestro recorrido por las distintas investigaciones que se están realizando, observamos la existencia de una serie de aspectos relacionados con los textos literarios que promueven interesantes propuestas de conocimiento transdiciplinar como sucede en el caso de Núñez Ramos (2017). Se revela también la existencia de áreas pendientes en la investigación neurocientífica educacional que se enumeran bajo el lema de nueva ciencia del aprendizaje (OECD, 2009). Las ideas que mencionamos a continuación se relacionan con estas áreas son esperanzadoras desde la perspectiva multidisciplinar. Cada una de ellas constituye un 
desafío para conseguir el apoyo financiero, académico y de procedimientos metodológicos que permitan la integración de profesionales de filología.

Los artículos de revisión consultados proponen nuevas líneas de investigación, a la vez que enmarcan la cronología de este proceso multidisciplinar. Además, algunos estudios de revisión abordan la relación en de la lingüística y las neurociencias, como el trabajo de Antoinette Hawayek (2010), donde se aporta una breve cronología del tema y se presenta la hipótesis de la arquitectura tripartita paralela, que intenta equiparar ambas ciencias, tanto a la lingüística, a partir de la teoría de la competencia, como la teoría de la actuación, adjudicada a la neurociencia.

Un informe interesante desde la perspectiva docente es el que se ha publicado sobre la nueva disciplina de neurodidáctica, ya que se plantea una serie bastante amplia de dimensiones que se pueden investigar en torno a la comunicación, el aprendizaje y la didáctica de la lengua aplicando resultados de investigaciones en neurobiología (Carrillo-García y Martínez-Ezquerro, 2018).

Se necesitan más investigaciones que profundicen en los múltiples beneficios de la literatura para el cerebro en distintos estadios de desarrollo del ser humano: en niños, jóvenes, adultos y ancianos, comparando situaciones de personas sanas, enfermas y con requerimientos específicos en su diario vivir.

Además, sería necesario comprender mejor cómo el ambiente influye en la adquisición de hábitos de lectura y de qué manera afecta la evolución intelectual de los lectores hasta convertirse en lectores expertos, comparando los distintos ambientes en relación con el desarrollo de los factores de comportamiento lector. El estudio de las expectativas y motivaciones de los estudiantes en torno a la lectura de textos literarios de buena calidad podría resultar de bastante interés.

Sería conveniente estudiar la literatura en relación con las emociones del cerebro humano para conocer de qué manera puede influir en la regulación emocional de niños y adolescen- 
tes, utilizando instrumentos psicológicos y de imagenología cerebral.

Un campo de trabajo fructífero y retador es el que se refiere a la confirmación de los beneficios de la lectura literaria usando métodos de investigación transdisciplinar, y cuyos resultados puedan ser reportados en revistas de estudios clínicos, por ejemplo, para estudiar las reservas cerebrales en las etapas longevas de desarrollo humano, para medir la resistencia al avance de enfermedades neurodegenerativas como el alzhéimer. En esa línea se sitúan también trabajos donde se evalúa el desarrollo lector de los estudiantes en relación con nutrición y parasitología, sobre todo en entornos vulnerables y de pobreza, como sucede por ejemplo, en la frontera de la República Dominicana con Haití.

Stanislas Dehaene establece un marco teórico robusto para el desarrollo de líneas de estudio dentro de lo que él ha denominado "la ciencia de la lectura". Nosotros, como profesionales de la filología, podemos aplicar las teorías y hallazgos de las neurociencias y comprometernos con investigaciones experimentales en torno a la literatura y la lengua en los distintos niveles de formación infantil, juvenil y universitaria. Esa nueva ciencia de la lectura no puede resolverlo todo, pero con la realización de proyectos viables que sigan los métodos adecuados se podrían evidenciar resultados probados y útiles para la comunidad científica.

En el caso de quien suscribe estas líneas los procesos de comprensión y expresión en lectura y escritura de los estudiantes dominicanos deben ser comprendidos a partir de su propia realidad; las soluciones que aportan los científicos son universales, sirven para cualquier estudiante en cualquier país, lo que nos permite trabajar en redes donde la colaboración aquilata el proceso de conocimiento. Nuestro compromiso consiste en trasladar esos conocimientos y desarrollar aplicaciones didácticas en el aprendizaje literario y de la lengua materna que reflejen los hallazgos de laboratorio divulgados por estos autores. 


\section{Referencias bibliográficas}

Alonso García, P. (2011), “El pensamiento narrativo. Aspectos cognitivos del relato, de Rafael Núñez Ramos", Teorema. Revista Internacional De Filosofía, 30, 2, 197-200.

Bid Educación (2016) Avances y desafios en educación en América Latina y El Caribe. Obtenido el 06/04/2019 en http://www. mendoza.edu.ar/wp-content/uploads/2017/03/avances-y-desaf\%C3\%ADos-en-Am\%C3\%A9rica-Latina-y-el-Caribe.pdf.

Bos, M. S., Vegas, E., Zoido, P. y Elias, A. (2016), Nota 3 América Latina y el Caribe en PISA en 2015: ¿Cuántos tienen bajo desempeño?, Washington DC, BID.

Carrillo-García, M. ${ }^{a}$ E. y Martínez-Ezquerro, A. (2018) "Neurodidáctica de la Lengua y la Literatura", Revista Iberoamericana de Educación, 78, 1, 149-164.

Castro, R. (2017) "Neurocientífico francés visitará el país", en Listín Diario. Obtenido el 20/11/2018 en https://listindiario. com/la-vida/2017/12/22/495975/.

Collège de France (s. f.) "Stanislas Dehaene: Experimental Cognitive Psychology“. Obtenido el 07/01/2019 en https://www. college-de-france.fr/site/en-stanislas-dehaene/.

Crespo, N., Benítez, R. y Pérez, L. (2010) “Conciencia metapragmática y la habilidad para producir narraciones escritas", Revista Signos, 43, 73, 179-209.

Dehaene, S. (2009) Reading in the brain, New York, Penguin.

Dehaene, S. (2015) Aprender a leer. De las ciencias cognitivas al aula, Buenos Aires, Siglo XXI.

Dehaene, S. (2017) El cerebro lector. Últimas noticias de las neurociencias sobre la lectura, la enseñanza, el aprendizaje y la dislexia, Buenos Aires, Siglo XXI, 3. ${ }^{\text {e ed. }}$.

Dehaene, S. (2018) "Aprender a aprender: curiosidad, libertad, educación y experimentación. Discurso de recepción del doctorado Honoris Causa de la Universidad Autónoma de Santo Domingo“, Enfoques, Investigación Lingüística y Literaria, 1, 1, 5054. 
Faust, M. y Kenett, Y. N. (2014) "Rigidity, chaos and integration: hemispheric interaction and individual differences in metaphor comprehension", Frontiers in Human Neuroscience, 8, Art. 511, 1-10.

Flotts, M. P., Manzi, J., Jiménez, D., Abarzúa, A., Cayuman, C., y García, M. J. (2015), Informe de resultados TERCE. Logros de aprendizaje. París-Santiago de Chile, UNESCO-OREALC/UNESCO-Santiago.

ForNAZZARI, L. (2008) “El papel del arte como protector de las funciones cerebrales. La música, la pintura y la escritura facilitan la capacidad de reserva cerebral“, Revista Mexicana de Neurociencia, 9, 2, 154-158.

Gómez Higuera, J. G., (2015) Utilización de integración del arte en los cuidados de enfermería, Tesis Doctoral, Universidad Complutense de Madrid.

González TApia, C. (2016) Neuroeducación y lingüística: una propuesta de aplicación a la enseñanza de la lengua materna, Tesis Doctoral, Universidad Complutense de Madrid.

HaWAyeK, A. (2010), "La lingüística en la neurociencia cognitiva: cerebro y lenguaje", en De la Mora Ochoa, A. (coord.), Rumbos de la lingüística. Lenguas amerindias, adquisición del lenguaje, relación lenguaje-cerebro, filología, México, Universidad Autónoma Metropolitana-Unidad Azcapotzalco, 139-161.

Kenett, Y. N., Gold, R. y Faust, M. (2018) "Metaphor comprehension in low and high creative individuals", Frontiers in Psychology, 9, Art. 482, 1-10.

Kidd, D. C. y Castano, E. (2013) "Reading literary fiction improves theory of mind", Science, 342 (6156), 377-380.

MARTín Jiménez, A. (2014) “La retórica clásica y la neurociencia actual: las emociones y la persuasión“, Rétor, 4, 1, 56-83.

Minerd (Ministerio de Educación de la República Dominicana) (2016a) Diseño curricular. Nivel primario, Santo Domingo, MINERD. 
Minerd (Ministerio de Educación de la República Dominicana) (2016b) Diseño curricular. Nivel secundario, Santo Domingo, MINERD.

Núñez Ramos, R. (2010) El pensamiento narrativo. Aspectos cognitivos del relato, Oviedo, Universidad de Oviedo.

Núñez Ramos, R. (2017) “La lectura como experiencia. Entre Aristóteles y la neuroestética“, Tropelias. Revista de Teoría de la Literatura y Literatura Comparada, Extr. 1, 221-235.

Oecd (Organización para la Cooperación y el Desarrollo Económicos) (2009) La comprensión del cerebro. El nacimiento de una ciencia del aprendizaje, Santiago de Chile, Universidad Católica Cardenal Raúl Silva Henríquez. Obtenido el 12/01/2019 en https://doi.org/10.1787/9789567947928-es.

Premack D. y Woodruff G. (1978) “Does chimpanzee have a theory of mind?", Behavior Brain Sciences, 4, 9-30.

Rodríguez, S. M. y Gallart, C. T. (2014) "Estimulación de la metacognición en la educación infantil mediante el lenguaje metafórico", International Journal of Developmental and Educational Psychology (Revista INFAD de Psicología), 6, 6, 511-518.

Tirapu-Ustárroz, J. Pérez-Sayes, G., Erekatxo-Bilbao, M. y Pelegrín-Valero, C. (2007) "¿Qué es la teoría de la mente?", Revista de Neurología, 44, 8, 479-489.

Unesco/Orealc (2015) Tercer Estudio Regional Comparativo y Explicativo (TERCE). Factores asociados, Santiago de Chile, OREALC/UNESCO Santiago».

Valdés, H., Treviño, E., Acevedo, C., Castro, M., Carrillo, S., Costilla, R., Bogoya, D. y Pardo, C. (2008), Segundo Estudio Regional Comparativo y Explicativo (SERCE). Los aprendizajes de los estudiantes de América Latina y el Caribe, Santiago de Chile, OREALC/UNESCO Santiago» 
